\title{
ОПРЕДЕЛЕНИЕ ЗНАЧИМЫХ ПОКАЗАТЕЛЕЙ ПЕРВИЧНОЙ ИНФОРМАЦИИ ДЛЯ СИСТЕМЫ С БИОЛОГИЧЕСКОЙ ОБРАТНОЙ СВЯЗЬЮ
}

\author{
В.И. Мещеряков ${ }^{1}$, Д.В. Мещеряков ${ }^{2}$, Е.В. Черепанова ${ }^{3}$ \\ ${ }^{1}$ Одесский государственный экологический университет, Украина \\ E-mail: ${ }^{1}$ gradan@ua.fm
}

Copyright $(\mathcal{C} 2017$ by author and the journal "Automation technological and business - processes". This work is licensed under the Creative Commons Attribution International License (CC BY). http://creativecommons.org/licenses/by/4.0/

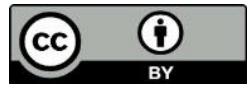

ONAFT

Open Access

Аннотация: Проанализированные реакциии человеческого организма при воздействии инфракрасного излучения различного спектрального диапазона применительно к разработке системь автоматического управления с биологической обратной связью. Показано, что информативность теплоотдачи человеческого организма может быть выражена ограниченным числом признаков: частотой сердечных сокращений и проводимостью кожи. Разработана методика испьтаний, экспериментальных исследований для выявления связи значимых показателей реакции человека с мощңностью инфракрасного облучения.

Abstract: There were analyzed reactions of the human body under the influence of infrared radiation of a different spectral range as applied to the development of an automatic control system with biological feedback. It was shown that the informative heat transfer of the human body can be expressed by a limited number of signs: heart rate and skin conductivity. A technique for testing and experimental research has been developed to identify the connection of significant indicators of human reaction with the power of infrared radiation.

Ключевые слова: инфракрасные излучатели, биологическая обратная связь, признаки, методика исследований.

Keywords: infrared radiators, biological feedback, symptoms, research methodology.

Введение

Системы с биологической обратной связью являются достаточно новым типом систем, в которых человек выступает не только средством принятия решений, но и объектом управления. Человеческий организм сам представляет собой крайне сложную систему, которую невозможно описать ограниченным признаковым пространством, доступным для обработки. В человеческом организме не существует признака, который бы являлся базовым (базовой системы координат), по отношению к которому можно рассматривать остальное признаковое пространство. Все признаки являются взаимосвязанными, неоднозначными, зависящими от внешних условий, состояния организма, предыстории процесса.

Вариантом такой системы с разомкнутой обратной связью является камера инфракрасной пелоидотерапии, предназначенной для лечения опорно-двигательного аппарата человека. Принцип действия инфракрасной пелоидотерапии состоит в замене египетского метода грязелечения с солнечным облучением на искусственное инфракрасное облучение, которое может быть реализовано в помещении [1]. При этом используются инфракрасные излучатели различного спектрального диапазона и специальная отражательная система, обеспечивающая бестеневое поле облучения пациента, что позволяет исключить влияние погоды, времени года и суток на результаты лечения. Система применяется для группового лечения с усредненными параметрами облучениями, определяемыми лечащим врачом в соответствии с характером заболевания, возрастом, полом, весом, накопленным опытом проведения аналогичных манипуляций и т.п.

Анализ литературных данных и постановка проблемы

Анализ целого ряда работ, например, [2-5], позволяет сделать вывод о том, что системы с биологической обратной связью оказываются эффективными в кардиологии при лечении детей, взрослых, тренировке летчиков при использовании электрокардиограмм в качестве значимого признака. Использование электроэнцефалограмм позволило успешно решать вопросы лечения эпилепсии [6], а совместное использование ЭКГ И ЭЭГ дало возможность создать систему биоуправления для преодоления патологического двигательного стереотипа [7]. Особо впечатляющие результаты по созданию систем с биологической обратной связью отмечены в психиатрии, например для тренинга 
http://www.atbp.onaft.edu.ua/

при тревожных расстройствах, резидентных к психофармакологии [8]. В тренажерах с биологической обратной связью выделяют однопараметрические и мультипараметрические системы [9], отличающиеся управлением различным числом функциональных показателей и их соотношением. Разработка структур информационных систем с биологической обратной связью в настоящее время находится в состоянии развития, поскольку цели систем разнятся, а признаковое пространство характеризуется неопределенностью и неоднозначностью [10].

Постановка задачи

Особенностью сложных систем является то обстоятельство, что признаки не одинаково значимы, большинство из них подстраивается под ограниченное число ведущих. Это свойство больших систем позволяет описывать их ограниченным числом значимых признаков или параметров порядка. При этом траектория системы в огромном фазовом пространстве состояний системы проецируется на подпространство меньшей размерности - русло [11]. Русло позволяет судить о системе в целом по этому пространству малой размерности. Система может иметь несколько русел. Подчиненные руслам признаковые пространства являются ведомыми и способствуют самоорганизации сложной системы. Поскольку в зависимости от состояния системы значимость отдельных признаков меняется, используется понятие области джокеров, меняющих степень неопределенности поведения системы [12]. В этой области система может случайным образом менять значимое признаковое пространство, описывающее ее поведение, что влияет на функции системы управления.

В медицинском приложении это особо важно, поскольку речь идет не о массовом использовании однотипных управляющих воздействий, а их приспособлении под конкретного индивидуума, набор признаков и технология воздействия для каждого пациента своя. В частности, для системы инфракрасной (ИК) пелоидотерапии [1] при групповом использовании бальнеологических процедур, оправдано использование усредненных показателей излучения ИК излучателей с дифференциацией для пожилых людей, людей среднего возраста и детей. При индивидуальном подходе к ИК пелоидотерапии необходимо подстраиваться под показатели данного индивидуума, т.е. его массу, возраст, сердечно-сосудистую систему, вид заболевания и т.п. Это делает пациента объектом управления, по считываемым показателям состояния которого должно осуществляться управление распределением интенсивности ИК поля. Для объекта управления необходимо выявить значащие признаки, манипуляция с которыми позволит формировать управляющие воздействия с заданной достоверностью.

Целью работы является определение необходимого числа признаков при воздействии инфракрасного излучения на человека, достаточного для обеспечения возможности управления интенсивностью облучения камерой пелоидотерапии, обеспечивающей комфортности пациента.

Для достижения этой цели необходимо решить следующие задачи:

1. проанализировать реакцию человека на статическое и динамическое инфракрасное излучение;

2. разработать методику проведения эксперимента для выявления значимых признаков, обеспечивающих возможность управления процессом биологической обратной связи.

Динамические показатели реакции человека на тепловое воздействие

При взаимодействии инфракрасного излучения с поверхностью кожи часть оптического излучения отражается, часть рассеивается и поглощается. Фотобиологические реакции возникают при поглощении электромагнитной энергии, которая возрастает с уменьшением длины волны. Различные слои кожи по-разному поглощают излучение длины волны. Глубина проникновения возрастает при переходе от ультрафиолетового до оранжевого от 0,7-0,8 до 2,5 мм, а для красного составляет 20-30 мм. В ближнем инфракрасном диапазоне на длине волны 950 нм проникающая способность достигает максимума и составляет 60-70 мм, в среднем и дальнем инфракрасном диапазонах резко снижается до 0,5-0,1 мм [13]. Коротковолновое инфракрасное излучение предпочтительно для внутреннего прогрева суставов, позвоночника, массажных манипуляций, длинноволновое - для общего прогрева поверхности тела. Процесс взаимодействия излучения различных спектральных диапазонов отличаются существенно. Длинноволновое передача поверхностной тепловой волны теплопроводностью, коротковолновое - объемное поглощение энергии теплового потока. При покрытии пелоидом участка тела глубинное проникновение излучения через пелоид невозможно.

Происходящее при поглощении энергии ИК излучения образование тепла приводит к локальному повышению температуры облучаемых кожных покровов на $1-2{ }^{\circ} \mathrm{C}$ и вызывает местные терморегуляционные реакции поверхностной сосудистой сети. Очевидно, что и отбор тепла за счет переноса крови будет более эффективен при объемном поглощении, поскольку сеть приповерхностных кровеносных сосудов также представляет собой объемное образование. Сосудистая реакция выражается в кратковременном спазме сосудов (до 30 сек), а затем увеличении локального кровотока и возрастании объема циркулирующей в тканях крови.

Важным параметром воздействия является время скрытой реакции на раздражитель - промежуток времени от момента раздражения до реакции на него. Время некоторых рефлекторных реакций имеет следующие значения [14]:

- на световое раздражение сетчатки $0,16-0,22$ сек.

- на слуховое раздражение $\quad 0,14-0,16$ сек.

- на болевое раздражение тепловое $0,36-0,40$ сек.

- на тепловое контактное раздражение $\quad 0,50-0,80$ сек.

- на холодное контактное раздражение $\quad 0,35-0,45$ сек.

Передача информации о наличии раздражителя поступает в центральную или периферическую нервную систему 
результирующая реакция на тепловое раздражение составляет 0,28-1,60 секунд.

Время скрытой реакции вегетативных процессов находится в диапазоне:

- глазно-сердечный рефлекс по изменению частоты пульса

- изменение частоты пульса в ответ на дозированную физическую нагрузку

- реакция потоотделения в ответ на дозированную физическую нагрузку

$5,2 \pm 0,3$ сек.

$1,2 \pm 0,1$ сек

$4,3 \pm 0,2$ сек.

- реакция расширения просвета сосудов в ответ на дозированную физическую нагрузку 7,8 $\pm 1,0$ сек.

- реакция сужения просвета сосудов в ответ на дозированную физическую нагрузку $\quad 8,9 \pm 0,9$ сек.

Теплоотдача избыточного тепла

Механизм терморегуляции человека как самоуправляющей системы включает две составляющие: физическую и химическую терморегуляции.

Основными видами теплоотдачи являются теплопроводность, конвекция, радиация и испарение.

Теплопроводность внешнего теплового потока во внутренний объем тела ограничена низкой теплопроводностью кожного покрова, а поскольку температура человеческого тела не может превышать $42^{\circ} \mathrm{C}$, снимаемый тепловой поток незначителен. Данный вид теплоотдачи можно отнести к пассивному методу, поскольку он практически не управляется центральной нервной системой. Измеряемыми показателями теплопроводности могут служить температура поверхностного слоя кожного покрова бесконтактными измерителями температуры, поскольку спектральный коэффициент черноты при максимуме излучения 8-10 мкм близок к единице.

Конвективная теплоотдача в основном обусловлена отбором тепла потоком крови в периферических областях близких к поверхности кожи. Данный вид отбора тепла является активным, управляется центральной нервной системой и проявляется в форме расширения диаметра сосудов до 6 раз и изменения скорости кровотока в $2-20$ раз при увеличении теплопроводность ткани [14]. Это позволяет распределять тепловой поток по всей поверхности тела и использовать большой коэффициент черноты и площадь кожного покрова человека для эффективного сброса избытка тепла радиационным излучением. Перераспределение потоков крови в организме может происходить практически без повышения частоты сердечных сокращений, если тепловые потоки незначительные или локальные. При повышении тепловых нагрузок повышается скорость кровотока за счет увеличения частоты сердечных сокращений. Измерение скорости кровотока в крупных сосудах осуществляется доплеровскими методами на ультразвуковых аппаратах, что практически исключает возможность их использование в качестве оперативных средств получения первичных признаков для управления излучением. Использование пульсоксиметров позволяет оперативно получать информацию о частоте сердечных сокращений, однако этот показатель характеризует лишь возможности перекачки крови сердечнососудистой системой, а не только связанный с этим отбор теплового потока с поверхности кожи.

Потоотделение является активным, управляемым центральной нервной системой, эффективным способом теплоотдачи, поскольку для испарения 1 г воды затрачивается 0,58 ккал, а при максимальном потоотделении от тела отводится примерно 870 ккал в час. При повышении температуры в камере пелоидотерапии до $35^{\circ} \mathrm{C}$ теплоотдача с помощью радиации и конвекции крайне ограничена и температура тела поддерживается на постоянном уровне испарением. Информацию о потоотделении получают обычно кондуктометрическим методом по изменению проводимости кожного покрова, поэтому он может быть рекомендован как средство оперативного контроля инфракрасного облучения.

Из приведенного качественного анализа следует, что частота сердечных сокращений является значимым признаком реакции организма на внешнее инфракрасное воздействие. Реакция сосудистой системы на отбор внешнего теплового потока кровеносной системой и начало работы системы сброса тепла испарением составляет примерно 10 секунд, что можно принять за предварительный показатель реакции на скачкообразное тепловое воздействие. Вторым значимым признаком реакции на плотность мощности инфракрасного излучения можно признать потоотделение, которое представляется проводимостью участка кожи.

Методика экспериментальных исследований

Динамические параметры используемых инфракрасных излучателей - определение времени запаздывания и постоянной времени при скачкообразном воздействии при нагреве и остывании излучателя.

Для пелоидотерапии могут использоваться инфракрасные излучатели следующих типов: керамические, трубчатые, ламповые, лазерные. Керамические излучатели мощностью 400-800 Вт генерируют длинноволновое излучение в диапазоне 6-12 мкм и представляют наиболее приемлемый вариант излучателей по критериям надежности и стабильности. Большая масса излучателя приводит к большим значениям времени запаздывания и постоянной времени. Трубчатые воздушные инфракрасные нагреватели мощностью 400-3000 Вт генерируют излучение в спектральном диапазоне 3-8 мкм, также обладают низкими динамическими характеристиками. Ламповые инфракрасные излучатели мощностью 100-300 Вт генерируют коротковолновое инфракрасное излучение в спектральном диапазоне 0,8-3,5 мкм и в связи с малой массой излучающего элемента имеют постоянную времени на уровне единиц секунд, что соизмеримо с динамическими характеристиками человека и значимо для управляющих систем. Углекислотный лазер с длиной волны 10,6 мкм имеют мощность излучения 100-1000 Вт и постоянную времени порядка единиц микросекунд, что существенно превышают динамические характеристики реакции человека, однако стоимость и надежность лазеров существенно превышает стоимость нагревательных инфракрасных излучателей. Динамические характеристики лазеров и ламповых инфракрасных излучателей меньше скрытой реакции вегетативных процессов человека, поэтому они могут быть рекомендованы для управляемых излучателей в системе с 
биологической обратной связью. В керамических и трубчатых излучателях время перехода из холодного в нагретое стационарное состояние при подаче питающего напряжения составляет единицы минут, поэтому они могут быть рекомендованы для установившихся или фоновых режимов работы облучателей в камере пелоидотерапии.

Реакция человека на П-образное инфракрасное воздействие - определение реакции организма на скачкообразное воздействие инфракрасного излучения и диапазона изменения контролируемых признаков, которые можно использовать как первичную информацию в системе с биологической обратной связью.

Контролируемые показатели: частота сердечных сокращений, сопротивление кожи, температура воздуха (закрытого от излучения датчика) в камере пелоидотерапии, температура поглощающей излучение пластины.

Методика определения передаточной функции человека для частоты сердечных сокращений (ЧСС) и проводимости кожи состоит в определении показателей частоты пульса пульсоксиметром и измерении сопротивления кожи при входе и выходе испытуемого в прогретую камеру пелоидотерапии. Перед входом в камеру испытуемые выдерживаются в течение времени установления стабильных показаний ЧСС. Фиксируется время входа в камеру пелоидотерапии и изменения показателей частоты и сопротивления с периодом, соответствующим теореме Котельникова, до их стабилизации. Выдерживаются стабильные показатели ЧСС и проводимости в течение примерно 100 периодов измерений. Фиксируется время выхода из камеры пелоидотерапии и измеряются показатели до стабилизации ЧСС и сопротивления кожи. По полученным результатам определяются время запаздывания, постоянные времени при нагреве и остывании, диапазоны изменений.

Реакция человека на трапецеидальное инфракрасное воздействие - определение реакции на плавное изменение мощности излучения инфракрасных излучателей до установившего значения ЧСС и проводимости кожи при нагреве и охлаждении.

Испытуемые входят в охлажденную камеру пелоидотерапии, и выдерживается время до стабилизации ЧСС. С фиксацией времени включаются все инфракрасные керамические излучатели, определяется ЧСС и проводимость до стабилизации показателей. Затем с фиксацией времени выключаются керамические излучатели, определяется изменение ЧСС и проводимость до остывания излучателей и стабилизации ЧСС. Нагрев излучателей определяют по температуре поверхности керамики термоэлектрическим датчиком.

Реакция человека на перепады мощунсти излучения - определение реакции человека на дозированные мощности инфракрасного облучения.

Испытуемые помещаются в непрогретую камеру пелоидотерапии и выдерживаются в течение времени до стабилизации ЧСС и сопротивления кожи. Включается $1 / 3$ излучателей и выдерживается время до стабилизации показателей на данном уровне мощности. Эксперимент повторяется для последовательно для 2/3 и $3 / 3$ мощности излучателей. Выключается группа излучателей до $2 / 3$ мощности и выдерживается время до стабилизации показателей. Эксперимент повторяется для снижения мощности излучения до $1 / 3$ и выключении всех излучателей до стабилизации ЧСС и сопротивления кожи. Данные позволяют судить о реакции организма на нагрев и остывание при одинаковых значениях мощности излучения.

Реакиия человека на затухающий гармонический процесс изменения мощности излучения - определение отображения частотой сердечных сокращений и проводимостью кожи изменения мощности инфракрасного облучения в диапазоне управления.

По датчику сопротивления кожи определяется уровень мощности излучения, соответствующий середине активной области диапазона датчика сопротивления. Обеспечивается возможность регулирования мощности быстродействующих излучателей относительного этого уровня в диапазоне примерно четверти общей мощности излучения. Устанавливается данный уровень излучения и выдерживается до стабилизации показаний датчика сопротивления кожи и ЧСС. Для имитации сходящегося процесса используется 8 ламп, которые обеспечивают нечетное количество уровней мощности, например, 9. После включения инерционных керамических излучателей и 4/8 ламповых излучателей должна быть обеспечена середина активной области датчика сопротивления. Испытуемые помещаются в камеру пелоидотерапии, и измеряется ЧСС и сопротивление кожи до установления стационарных значений. С периодом 2 минуты включаются $5 / 8$ ламповых излучателей, фиксируется ЧСС и сопротивление кожи; 6/8; 7/8; 8/8; 7/8; 6/8; 5/8; 4/8; 3/8; 2/8; 1/8; 0/8; 1/8; 2/8; 3/8; 4/8; 5/8; 6/8; 7/8; 6/8; 5/8; 4/8; 3/8; 2/8; 1/8; 2/8; 3/8; 4/8; 5/8; $6 / 8 ; 5 / 8 ; 4 / 8 ; 3 / 8 ; 2 / 8 ; 3 / 8 ; 4 / 8 ; 5 / 8 ; 4 / 8 ; 3 / 8 ; 4 / 8 ; 4 / 8 ; 4 / 8$. Эксперимент повторяется для периода 60, 30, 20 секунд.

Реакиия человека на пульсирующее коротковолновое инфракрасное излучение - определение связи чСС и сопротивления кожи с импульсным коротковолновым инфракрасным излучением различной периодичности.

Эксперимент проводится в прогретой камере в лежачем положении пациента на кушетке, над которой расположены ламповые инфракрасные источники, излучение которых направлено на позвоночник. Облучение пациента происходит в течение 10 минут с периодом колебаний 5, 10, 20, 30, 60 секунд со скважностью 2 . Контроль осуществляется по ЧСС и сопротивлению кожи.

Выводы

1. Проведен анализ согласования реакции человека с динамическими характеристиками инфракрасных излучателей и выделены типы излучателей, которые могут быть использованы для реализации систем с биологической обратной связью.

2. Разработана методика проведения экспериментальных исследований для выявления связи значимых показателей реакции человека с мощностью инфракрасного облучения как обратная задача управления. 
Литература

[1] Деклараційний патент № 58051А (Україна). Спосіб пелоїдотерапії та камера для його здійснення / Косовєров Є.О., Тищук М.М., Мещеряков В.І., Веселкова Т.О.

[2] Hallman, D.M. Effects of heart rate variability biofeedback in subjects with stress - related chronic neck pain: a pilot study / D.M. Hallman, E.M. Olsson, D. Von Scheele // Appl. Psychophysiol. Biofeedback. 2011. Vol. 36, N. 2. P. $71-$ 80.

[3] Andrasic F. Biofeedback in headache: An overview of approaches and evidence / Andrasic F. // Cleveland Clinic Journal of Medicine. 2010. Vol. 77, Suppl. 3. S. 72-76.

[4] Nada, P.J. Heart rate variability in the assessment and biofeedback training of common mental health problems in children / P.J. Nada // Med. Arh. 2009. Vol. 63, N. 5. P. 244-248.

[5] Wheat, A.L. Biofeedback of heart rate variability and related physiology: a critical review / A.L. Wheat, K.T. Larkin // Appl. Psychophysiol. Biofeedback. 2010. Vol. 35, N. 3. P. 229-242.

[6] Sterman, M. B. EEG biofeedback in the treatment of epilepsy: An overview circa 1980. In: Clinical Biofeedback: Efficacy and Mechanism / M. B. Sterman // Guilford, NY, 1982, P. 330-331,

[7] Циркин, Г. М. К вопросу о применении ЭЭГ- и ЭМГ-биоуправления для преодоления патологического двигательного стереотипа / Г. М. Циркин, М. М. Шперлинг // Бюллетень Сибирского отделения Российской академии медицинских наук, 2004, № 3, с. 132-133.

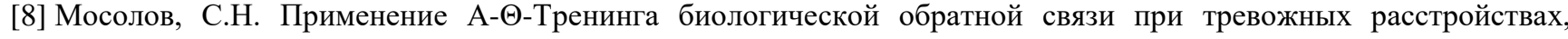
резистентных к психофармакотерапии (открытое рандомизированное контролируемое исследование) / С.Н. Мосолов, Е.В. Бирюкова, И.В. Тимофеев // Обозрение психиатрии и медицинской психологии им. В.М. Бехтерева. 2010. №1, с.15-20.

[9] Пятокович, Ф.А. Мультипараметрическая одноканальная система управления в игровом модуле «Хотх» с биологической обратной связью / Ф.А. Пятокович, М. А. Сурушкин // Фундаментальные исследования. - 2011. - №4. - С. 139-144.

[10] Сороко, С.И. Нейрофизиологические и психофизио-логические основы адаптивного биоуправления / С.И. Сороко, В.В. Трубачев // СПб.: ИЭФБ РАН, 2010. - 607 с.

[11] Котов, Ю.Б. Новые математические подходы к задачам медицинской диагностики / Ю.Б. Котов // М.: Едиториал УРСС, 2004. - 328 с.

[12] Чернавский, Д.С. Синергетика и информация / Д.С. Чернавский // М.: УРСС, 2004. - 288 с.

[13] Справочная книга по светотехнике. Под ред. Ю.Б. Айзенберга. М.: Знак, 2006. - 972 с.

[14] Зайчик, А.Ш. Патологическая физиология. Том 2 / А.Ш. Зайчик, Л.П. Чурилов // СПб.: ЭЛБИ-СПб, 2007. $768 \mathrm{c.}$

\section{References}

[1] Declarative Patent No. 58051A (Ukraine). The method of peloidotherapy and a camera for its implementation / Kosovyrov Ye.O., Tyshchuk M.M., Meshcheryakov V.I., Veselkova T.O.

[2] Hallman D.M., Olsson E.M., Von Scheele D. (2011). Effects of heart rate variability on biofeedback in subjects with stress-related chronic neck pain: a pilot study. Appl. Psychophysiol Biofeedback, 36, 2, 71-80.

[3] Andrasic F. (2010). Biofeedback in headache: An overview of approaches and evidence. Cleveland Clinic Journal of Medicine, 77, 3, 72-76.

[4] Nada P.J. (2009). Heart rate variability in the assessment and biofeedback training for common mental health problems in children. Med. Arh, 63, 5, 244-248.

[5] Wheat A.L., Larkin K.T. (2010). Biofeedback of heart rate variability and related physiology: a critical review. Appl. Psychophysiol Biofeedback, 35, 3, 229-242.

[6] Sterman M. B. (1982). EEG Biofeedback in the Treatment of Epilepsy: An overview circa 1980. In: Clinical Biofeedback: Efficacy and Mechanism. Guilford, NY, 330-331.

[7] Tsirkin G.M., Shperling M.M. (2004). On the use of EEG and EMG biofeedback for overcoming the pathological motor stereotype. Bulletin of the Siberian Branch of the Russian Academy of Medical Sciences, 3, 132-133.

[8] Mosolov S.N., Biryukova E.V., Timofeev I.V. (2010). Application of A- $\Theta$-Training of biological feedback in case of anxiety disorders resistant to psychopharmacotherapy (open randomized controlled trial). Survey of psychiatry and medical psychology. V.M. Bechterew, 1, 15-20.

[9] Pyatokovich F.A., Surushkin M.A. (2011). Multiparametric single-channel control system in the game module "Hoth" with biological feedback. Fundamental Research, 4, 139-144.

[10] Soroko S.I., Trubachev V.V. (2010). Neurophysiological and psychophysiological foundations of adaptive biofeedback. St. Petersburg: IEPB RAS, 607.

[11] Kotov Yu.B. (2004). New mathematical approaches to the problems of medical diagnostics. Moscow: Editorial URSS, 328.

[12] Chernavsky D.S. (2004). Synergetics and information. M .: URSS, 288.

[13] Ed. Eisenberg Yu.B. (2006). Reference book on lighting equipment. M.: Sign, 972.

[14] Zaichik A.Sh., Churilov L.P. (2007). Pathological physiology. Volume 2. SPb.: ELBI-SPb, 768. 